\title{
High-Pitch Computed Tomography Coronary Angiography-A New Dose-Saving Algorithm: Estimation of Radiation Exposure
}

\author{
Dominik Ketelsen, ${ }^{1}$ Markus Buchgeister, ${ }^{2}$ Andreas Korn, ${ }^{1}$ Michael Fenchel, ${ }^{3}$ \\ Bernhard Schmidt, ${ }^{4}$ Thomas G. Flohr, ${ }^{4}$ Christoph Thomas, ${ }^{1}$ Christoph Schabel, ${ }^{1}$ \\ Ilias Tsiflikas, ${ }^{1}$ Roland Syha, ${ }^{1}$ Claus D. Claussen, ${ }^{1}$ and Martin Heuschmid ${ }^{1}$ \\ ${ }^{1}$ Department of Diagnostic and Interventional Radiology, University Hospital of Tübingen, Hoppe-Seyler-Straße 3, \\ 72076 Tübingen, Germany \\ ${ }^{2}$ Department of Mathematics, Physics \& Chemistry, Beuth University of Applied Sciences, Luxemburger Straße 10, \\ 13353 Berlin, Germany \\ ${ }^{3}$ Department of Diagnostic and Interventional Neuroradiology, University Hospital of Tübingen, Hoppe-Seyler-Straße 3, \\ 72076 Tübingen, Germany \\ ${ }^{4}$ Division of Computed Tomography, Siemens Medical Solutions, An der Lände 1, 91301 Forchheim, Germany
}

Correspondence should be addressed to Dominik Ketelsen, dominik.ketelsen@med.uni-tuebingen.de

Received 2 March 2012; Accepted 4 April 2012

Academic Editor: Andreas H. Mahnken

Copyright (c) 2012 Dominik Ketelsen et al. This is an open access article distributed under the Creative Commons Attribution License, which permits unrestricted use, distribution, and reproduction in any medium, provided the original work is properly cited.

Purpose. To estimate effective dose and organ equivalent doses of prospective ECG-triggered high-pitch CTCA. Materials and Methods. For dose measurements, an Alderson-Rando phantom equipped with thermoluminescent dosimeters was used. The effective dose was calculated according to ICRP 103. Exposure was performed on a second-generation dual-source scanner (SOMATOM Definition Flash, Siemens Medical Solutions, Germany). The following scan parameters were used: $320 \mathrm{mAs}$ per rotation, 100 and $120 \mathrm{kV}$, pitch 3.4 for prospectively ECG-triggered high-pitch CTCA, scan range of $13.5 \mathrm{~cm}$, collimation $64 \times 2 \times 0.6 \mathrm{~mm}$ with z-flying focal spot, gantry rotation time $280 \mathrm{~ms}$, and simulated heart rate of 60 beats per minute. Results. Depending on the applied tube potential, the effective whole-body dose of the cardiac scan ranged from $1.1 \mathrm{mSv}$ to $1.6 \mathrm{mSv}$ and from 1.2 to $1.8 \mathrm{mSv}$ for males and females, respectively. The radiosensitive breast tissue in the range of the primary beam caused an increased female-specific effective dose of $8.6 \% \pm 0.3 \%$ compared to males. Decreasing the tube potential, a significant reduction of the effective dose of $35.8 \%$ and $36.0 \%$ can be achieved for males and females, respectively $(P<0.001)$. Conclusion. The radiologist and the CT technician should be aware of this new dose-saving strategy to keep the radiation exposure as low as reasonablly achievable.

\section{Introduction}

At present, computed tomography coronary angiography (CTCA) is an important, widely accepted diagnostic tool for the assessment of coronary artery disease. Several studies have shown the potential of different dose-saving strategies to keep the radiation exposure as low as reasonablly achievable. Hausleiter et al. reported in an international multicenter trial (PROTECTION I) a mean effective dose of $12 \mathrm{mSv}$ in CTCA, ranging from 5 to $30 \mathrm{mSv}$ [1]. Radiation exposure can be reduced substantially by currently available strategies, but these possibilities are used infrequently [1].

Since the introduction of a second-generation dualsource scanner system, a new scanning mode with increased table feed is available. Compared to retrospective ECGgated and prospective ECG-triggered CTCA, this highpitch, prospective triggered scanning mode has the potential for drastic dose reduction due to a gapless imaging of the heart within one heartbeat with no overlapping data acquisition. 
The aim of the study was to estimate effective wholebody dose and organ equivalent doses of prospective ECGtriggered high-pitch CTCA.

\section{Material and Methods}

2.1. Dosimetry. The experiments were performed by using an anthropomorphic, hermaphrodite male phantom with breast phantom attachments (Alderson-Rando phantom; Alderson Research Laboratories Inc., Stanford, CT, USA). The phantom was equipped with 117 thermoluminescent dosimeters (TLDs) with dimension of $1 \times 1 \times 6 \mathrm{~mm}$ (TLD$100 \mathrm{H}$, Bicon-Harshow, Radiation Measurement Products, Cleveland, $\mathrm{OH}, \mathrm{USA}$ ) to perform radiation exposure measurements [2-4].

By using a Philips Optimus 65 (Philips Medical Systems, PC Best, The Netherlands), a calibration of the 117 was calculated, which was defined by means of parallel exposure of 33 TLDs with a known radiation dose using $102 \mathrm{kV}$ and $10 \mathrm{~mA}$ for $100 \mathrm{~ms}$ at a source-skin distance (SSD) of $100 \mathrm{~cm}$. To minimize the Heel effect, wire markers in the field were avoided and all expositions were done in the same position with respect to the orientation of the X-ray tube. Crosschecked by an ionization dosimeter positioned in the same phantom depths, the reference TLDs were exposed with a dose of $1.081 \mathrm{mGy}$. No further correction factors were used as the calibration voltage is close to the CT tube voltage.

The evaluation of the irradiated TLDs was performed using a TLD reader (Model 5500 TLD Reader, Bicron Radiation Measurement Products, Solon, OH, USA) within $24 \mathrm{~h}$ after radiation exposure. The readout TLD values in nanocoulombs were multiplied by an individual calibration factor.

Dependent on the anatomical position of each organ 39 different positions in the Alderson-Rando Phantom were used to assess the organ doses. Due to measurement deviation, three TLDs were placed at each point of dose measurement to minimize bias. The number of TLDs allocated to different organ positions were as follows: 3 at the brain, thyroid gland, esophagus, thymus, heart, breast, stomach, upper colon, spleen, kidneys, adrenal glands, pancreas, small intestine, lower colon, urinary bladder, muscle tissue, red bone marrow, skin, ovaries, and testicles, 42 at the lung, and 15 at the liver.

The effective dose was calculated by summarizing the weighted organ doses according to the guidelines of ICRP 103 [5]. Radiation doses of simulated small organs (i.e., thyroid gland) were directly rated into the calculation. Doses of larger organs (i.e., lung) were determined by assessing the mean of measured TLDs from the entire organ.

To assess gender-specific differences, the testicles were used to measure the male-specific gonadal dose while radiation dose of the breast and the ovaries accounted to the female-specific radiation exposure. The breast phantom attachments could influence the calculation of the malespecific effective dose by increased soft tissue in the scan range. Nevertheless, evaluated values of effective dose using a hermaphrodite phantom are sufficiently precise for application in radiological radiation protection $[5,6]$.
2.2. Scan Protocols. Scans were performed on a secondgeneration dual-source CT scanner (SOMATOM Definition Flash, Siemens Medical Solutions, Forchheim, Germany). The following parameters were used: $320 \mathrm{mAs}$ per rotation, 100 and $120 \mathrm{kV}$, pitch 3.4 for prospectively ECG-triggered CTCA (table feed $450 \mathrm{~mm} / \mathrm{s}$ ), scan range of $13.5 \mathrm{~cm}$ (four blocks of sequential detector coverage), collimation $64 \times 2 \times$ $0.6 \mathrm{~mm}$ with $\mathrm{z}$-flying focal spot, and gantry rotation time $280 \mathrm{~ms}$. The scanner software simulated a heart rate of 60 beats per minute. $60 \%$ of the RR-interval was set to trigger the scan. In high-pitch CTCA, the heart is scanned within one heartbeat. Data acquisition with two X-ray tubes and detectors allows a gapless imaging of the heart despite a high pitch of 3.4. Gaps in the data from the first measurement system resulting from the high pitch are filled completely with the data from the second measurement system that acquires the data a quarter rotation later [7].

2.3. Statistical Analysis. Normal distribution of measured organ-specific dose values of each scan protocol was assessed using the Kolmogorov-Smirnov test. Statistical significance between different scan protocols was evaluated using the two-tailed paired Student's $t$-test comparing the measured organ-specific dose values. A $P$ value $<0.05$ was considered to be significant.

\section{Results}

Effective, gender-specific doses of scan protocols with $100 \mathrm{kV}$ and $120 \mathrm{kV}$ and reported CT dose index and dose length product are shown in Table 1.

Depending on the applied tube potential, the effective whole-body dose of the cardiac scan ranged from $1.1 \mathrm{mSv}$ to $1.6 \mathrm{mSv}$ and from 1.2 to $1.8 \mathrm{mSv}$ for males and females, respectively.

Directly irradiated organs within the scan range received organ equivalent doses of up to $0.33 \mathrm{mSv}$ to the lung and up to $0.13 \mathrm{mSv}$ to the breast. The radiosensitive breast tissue in the range of the primary beam caused an increased femalespecific effective dose of $8.6 \% \pm 0.3 \%$ compared to males. The gonadal doses were $<0.02 \mathrm{mSv}$ in both scan protocols. Details about organ equivalent doses are displayed in Table 2.

A reduction of the tube potential from $120 \mathrm{kV}$ to $100 \mathrm{kV}$ significantly reduces the radiation exposure by $35.8 \%$ for males and $36.0 \%$ for females, respectively $(P<0.001)$.

\section{Discussion}

Several studies described the correlation between the pitch value and the radiation exposure in computed tomography coronary artery (CTCA). Typically, the pitch is less than 1 , usually between 0.2 and 0.5 in first-generation dualsource CTCA resulting in relevant overlaps by advancing the table much less than one detector width during one scanner rotation $[3,7]$. Thus, repeated exposure of the same heart region during several consecutive rotations causes an increase of radiation exposure.

The evaluated, prospective ECG-triggered high-pitch CTCA uses a pitch value of 3.4 resulting in a table feed of 
TABLE 1: Effective radiation exposure of high-pitch CTCA using $100 \mathrm{kV}$ and $120 \mathrm{kV}$.

\begin{tabular}{lccccc}
\hline Protocol & $\begin{array}{c}\text { Effective dose } \\
\text { male }[\mathrm{mSv}]\end{array}$ & $\begin{array}{c}\text { Effective dose } \\
\text { female }[\mathrm{mSv}]\end{array}$ & Total mAs & CTDIVol $^{(\mathrm{mGy})}$ & DLP (mGy cm) \\
\hline $1(100 \mathrm{kV})$ & 1.1 & 1.2 & 688 & 3.1 & 56 \\
$2(120 \mathrm{kV})$ & 1.6 & 1.8 & 689 & 5.2 & 95 \\
\hline
\end{tabular}

$\mathrm{CTDI}_{\mathrm{Vol}}$ : volume CT dose index; DLP: dose-length product.

TABLe 2: Measured organ equivalent doses of high-pitch CTCA using different tube voltages ( $\mathrm{mSv})$.

\begin{tabular}{lcc}
\hline Organ/tissue & $100 \mathrm{kV}$ & $120 \mathrm{kV}$ \\
\hline Thyroid gland & 0.03 & 0.04 \\
Esophagus & 0.05 & 0.05 \\
Lung & 0.20 & 0.33 \\
Breast & 0.07 & 0.13 \\
Stomach & 0.45 & 0.67 \\
Liver & 0.06 & 0.12 \\
Colon & 0.03 & 0.03 \\
Urinary bladder & 0.00 & 0.00 \\
Red bone marrow & 0.01 & 0.02 \\
Skeleton & 0.00 & 0.00 \\
Skin & 0.00 & 0.01 \\
Male gonads & 0.00 & 0.00 \\
Female gonads & 0.01 & 0.01 \\
Remaining organs & 0.22 & 0.37 \\
\hline
\end{tabular}

$450 \mathrm{~mm} / \mathrm{s}$ with no overlapping data acquisition by filling the gaps in the data of the spiral acquisition of the first measurement system with the data from the second measurement system one-quarter rotation later. The whole heart is completely scanned within one heart cycle, that is, the topmost image will be acquired at the set trigger point and subsequent images display the heart in later phases of the heart cycle [8-11].

In this study, we determined the effective radiation exposure of prospective ECG-triggered high-pitch CTCA depending on the gender and the used tube voltage. The results are concordant to recently reported dose values from Goetti et al. [8]. The radiation exposure is substantially lower than other reported dose values of retrospective ECG-gated or prospective ECG-triggered CTCA. A comparative study of different dose-saving techniques reports effective dose values of $5.8 \mathrm{mSv}$ to $16.0 \mathrm{mSv}$ for retrospective ECG-gated protocols without ECG-pulsing, and $4.2 \mathrm{mSv}$ to $9.8 \mathrm{mSv}$ for protocols with different ECG-pulsing techniques and $2.8 \mathrm{mSv}$ to $4.3 \mathrm{mSv}$ for prospective ECG-triggered, the socalled step-and-shoot, CTCA [12].

A further radiation protective effect comprises the acquisition of less overlapping data. This results in reduction of the organ equivalent dose of the extremely radiosensitive female breast tissue, which is always in the scan range, but rarely organ of interest. In high-pitch CTCA, the femalespecific effective dose is higher compared to male effective dose measurements by an average of up to $8.6 \%$. In conventional overlapping CTCA data acquisition, however, females received increased effective dose values of up to $70 \%[6,12-$ $14]$.

By decreasing the tube voltage, the effective dose can be further reduced by up to $36.0 \%$ in high-pitch CTCA. However, changes in tube voltage are complex and affect image noise as well as tissue contrast. Nevertheless, several studies confirmed a sufficient image quality of $100 \mathrm{kV}$ CTCA protocols in slender patients with a body mass index lower than $30 \mathrm{~kg} / \mathrm{m}^{2}[15]$.

A possible limitation of the study is the lack to determine image quality as an additional parameter of the technique. However, initial feasibility studies reported an excellent image quality in a selected patient population $[9,15]$. Highpitch CTCA shows equivalent image quality in patients with low and stable heart rates up to $60 \mathrm{bpm}$ compared to prospective ECG-triggered and retrospective ECG-gated CTCA. A diagnostic image quality can be achieved in $97 \%$ of coronary segments with high-pitch CTCA [16-18]. These studies recommend this prospective, R-wave triggered, highpitch scanning mode for patients with low and regular heart rates $(<55-60$ beats per minute) to acquire the complete dataset before the onset of atrial contraction $[7,9,10]$.

\section{Conclusion}

In conclusion, the prospective ECG-triggered high-pitch CTCA has the potential to scan the heart with an effective dose between $1 \mathrm{mSv}$ and $2 \mathrm{mSv}$, which is substantially lower than previously described CTCA scanning modes. The radiologist and the CT technician should be aware of this new dose-saving strategy to keep the radiation exposure as low as reasonablly achievable.

\section{Disclosure}

B. Schmidt and T. G. Flohr are employees of Siemens Medical Solutions.

\section{References}

[1] J. Hausleiter, T. Meyer, F. Hermann et al., "Estimated radiation dose associated with cardiac CT angiography," Journal of the American Medical Association, vol. 301, no. 5, pp. 500-507, 2009. 
[2] D. Ketelsen, M. H. Luetkhoff, C. Thomas et al., "Estimation of the radiation exposure of a chest pain protocol with ECGgating in dual-source computed tomography," European Radiology, vol. 19, no. 1, pp. 37-41, 2009.

[3] D. Ketelsen, C. Thomas, M. Werner et al., "Dual-source computed tomography: estimation of radiation exposure of ECG-gated and ECG-triggered coronary angiography," European Journal of Radiology, vol. 73, no. 2, pp. 274-279, 2010.

[4] P. Hunold, F. M. Vogt, A. Schmermund et al., "Radiation exposure during cardiac CT: effective doses at multi-detector row CT and electron-beam CT," Radiology, vol. 226, no. 1, pp. 145152, 2003.

[5] "The 2007 recommendations of the international commission on radiological protection," Annals of the ICRP, vol. 37, no. 24, pp. 1-332, 2007, ICRP publication 103.

[6] H. Von Boetticher, J. Lachmund, H. K. Looe, W. Hoffmann, and B. Poppe, "2007 Recommendations of the ICRP change basis for estimation of the effective dose: what is the impact on radiation dose assessment of patient and personnel?" RoFo Fortschritte auf dem Gebiet der Rontgenstrahlen und der Bildgebenden Verfahren, vol. 180, no. 5, pp. 391-395, 2008.

[7] S. Achenbach, M. Marwan, T. Schepis et al., "High-pitch spiral acquisition: a new scan mode for coronary CT angiography," Journal of Cardiovascular Computed Tomography, vol. 3, no. 2, pp. 117-121, 2009.

[8] R. Goetti, S. Leschka, M. Boschung et al., "Radiation doses from phantom measurements at high-pitch dual-source computed tomography coronary angiography," European Journal of Radiology, vol. 81, no. 4, pp. 773-779, 2012.

[9] M. Lell, M. Marwan, T. Schepis et al., "Prospectively ECGtriggered high-pitch spiral acquisition for coronary CT angiography using dual source CT: technique and initial experience," European Radiology, vol. 19, no. 11, pp. 2576-2583, 2009.

[10] T. G. Flohr, E. Klotz, T. Allmendinger, R. Raupach, H. Bruder, and B. Schmidt, "Pushing the envelope: new computed tomography techniques for cardiothoracic imaging," Journal of Thoracic Imaging, vol. 25, no. 2, pp. 100-111, 2010.

[11] T. G. Flohr, S. Leng, L. Yu et al., "Dual-source spiral CT with pitch up to 3.2 and $75 \mathrm{~ms}$ temporal resolution: image reconstruction and assessment of image quality," Medical Physics, vol. 36, no. 12, pp. 5641-5653, 2009.

[12] D. Ketelsen, M. Fenchel, M. Buchgeister et al., "Estimation of radiation exposure of different dose saving techniques in 128slice computed tomography coronary angiography," European Journal of Radiology, vol. 81, no. 2, pp. e153-e157, 2012.

[13] L. W. Poll, M. Cohnen, S. Brachten, K. Ewen, and U. Mödder, "Dose reduction in multi-slice CT of the heart by use of ECG-controlled tube current modulation ("ECG pulsing"): phantom measurements," RoFo Fortschritte auf dem Gebiet der Rontgenstrahlen und der Bildgebenden Verfahren, vol. 174, no. 12, pp. 1500-1505, 2002.

[14] T. Trabold, M. Buchgeister, A. Kuttner et al., "Estimation of radiation exposure in 16-detector row computed tomography of the heart with retrospective ECG-gating," RoFo Fortschritte auf dem Gebiet der Rontgenstrahlen und der Bildgebenden Verfahren, vol. 175, no. 8, pp. 1051-1055, 2003.

[15] S. Leschka, P. Stolzmann, L. Desbiolles et al., "Diagnostic accuracy of high-pitch dual-source CT for the assessment of coronary stenoses: first experience," European Radiology, vol. 19, no. 12, pp. 2896-2903, 2009.

[16] J. R. Ghadri, S. M. Kuest, R. Goetti et al., "Image quality and radiation dose comparisonof prospectively triggered low-dose
CCTA: 128-slice dual-source high-pitch spiral versus 64slice single-source sequential acquisition," The International Journal of Cardiovascular Imaging. In press.

[17] L. A. Neefjes, A. S. Dharampal, A. Rossi et al., "Image quality and radiation exposure using different low-dose scan protocols in dual-source CT coronary angiography: randomized study," Radiology, vol. 261, no. 3, pp. 779-786, 2011.

[18] P. Stolzmann, R. P. Goetti, P. Maurovich-Horvat et al., "Predictors of image quality in high-pitch coronary CT angiography," American Journal of Roentgenology, vol. 197, no. 4, pp. 851$858,2011$. 


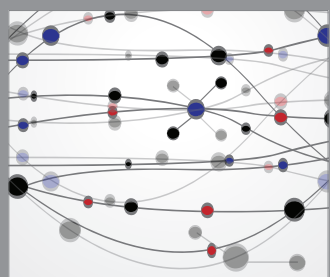

The Scientific World Journal
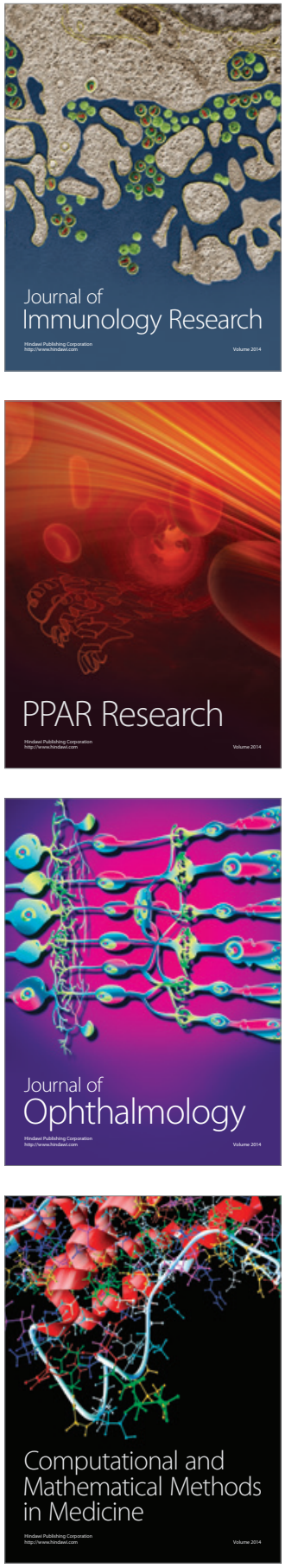

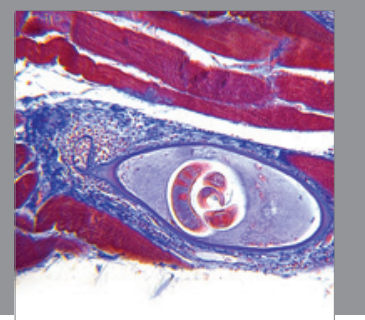

Gastroenterology

Research and Practice
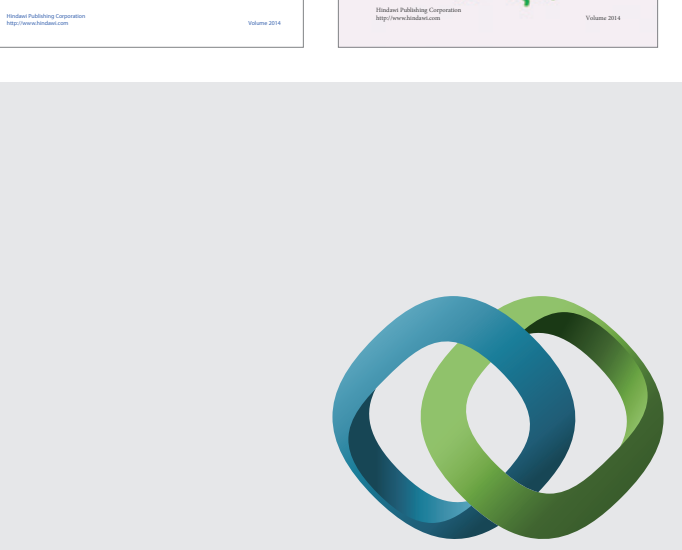

\section{Hindawi}

Submit your manuscripts at

http://www.hindawi.com
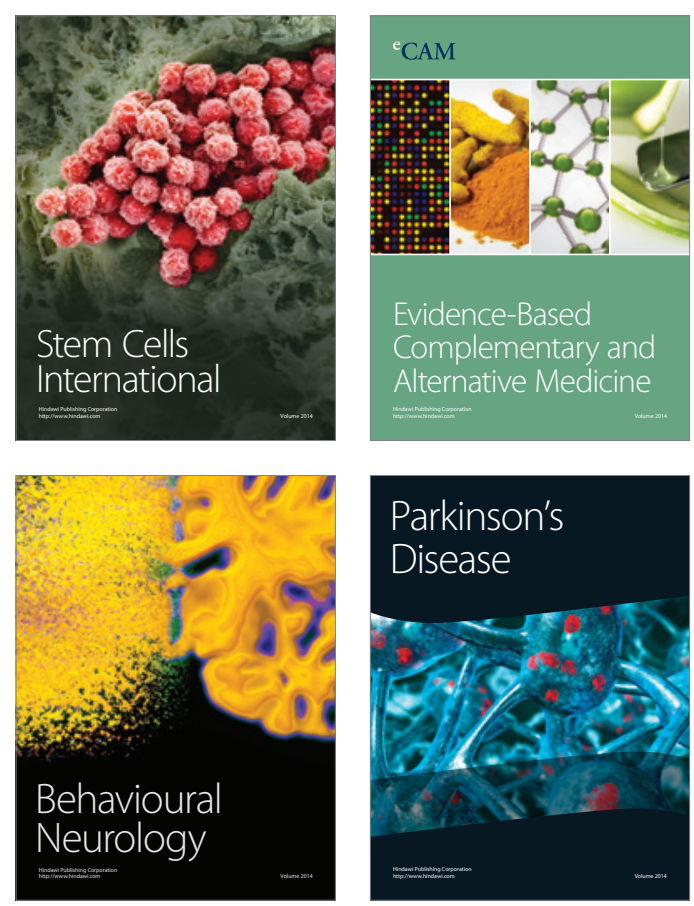

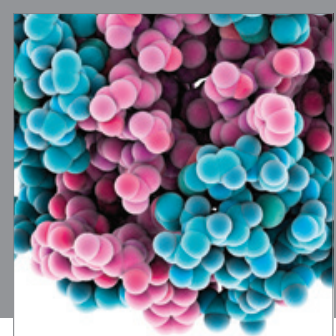

Journal of
Diabetes Research

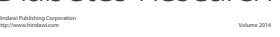

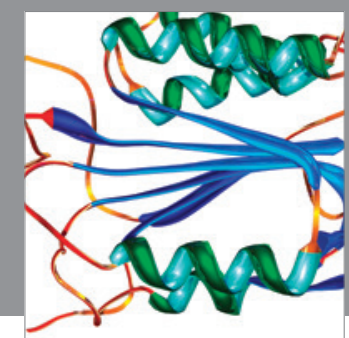

Disease Markers
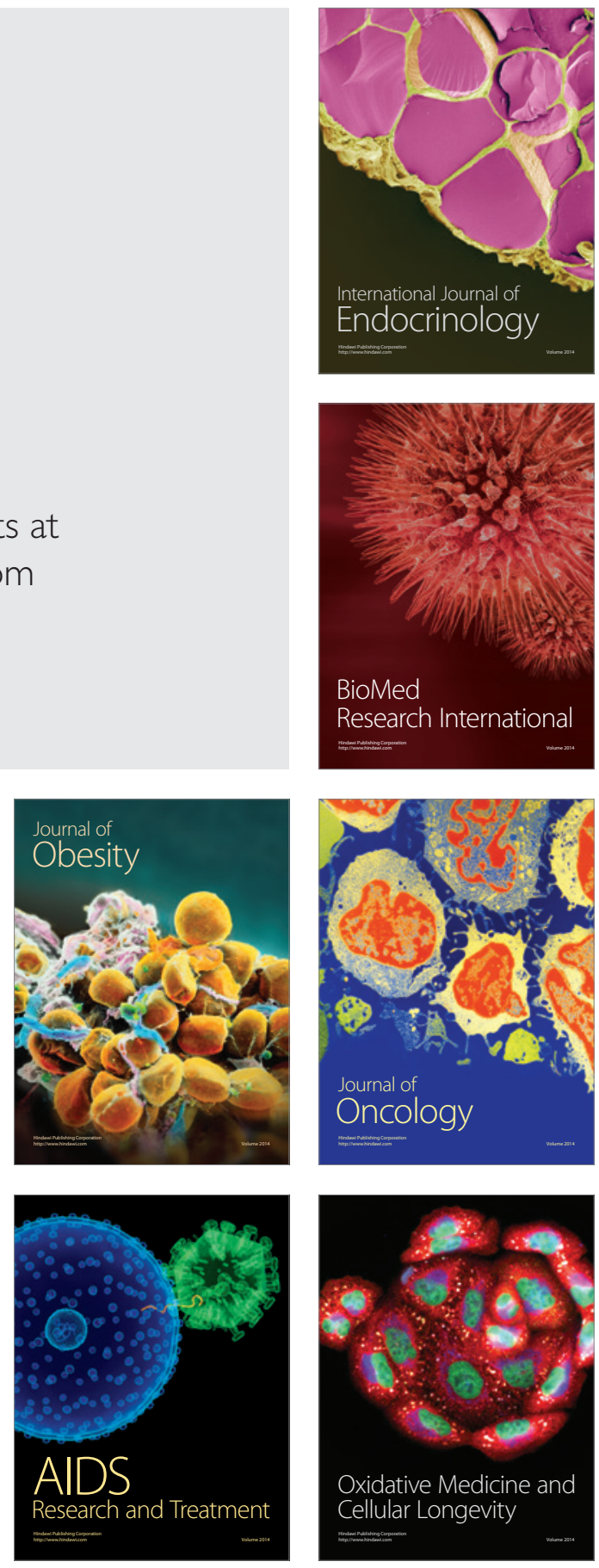